$\mathbb{P}$ periodica polytechnica

Transportation Engineering

$41 / 1(2013) 71776$

doi: $10.3311 /$ PPtr.7103

http://periodicapolytechnica.org/tr

Creative Commons Attribution (i)

RESEARCH ARTICLE

\section{Enhancement of Driver Speed Based on Multi-Criteria Optimization}

\author{
András Mihály / Balázs Németh / Péter Gáspár
}

Received 2012-11-13

\begin{abstract}
The paper focuses on the design of an adaptive cruise control system which follows the behavior of the driver besides the optimization of longitudinal energy and fuel consumption. By using road information about the future characteristics of the road, e.g. oncoming speed limits or road slopes, it is possible to modify the speed during the journey in advance. The aim is to enhance the comfort of the driver and the passengers by adopting the natural behavior of the driver in the speed selection process and at the same time road information is taken into consideration. The driver behavior is captured using a longitudinal driver model. The main novelty of the paper is the incorporation of the driver behavior in the look-ahead control algorithm. It is demonstrated in real data simulation that with the proposed method a significant amount of fuel can be saved while the speed profile is closer to that of the human driver.
\end{abstract}

\section{Keywords}

driver model $\cdot$ look-ahead control $\cdot$ multi-criteria optimization optimal velocity

\section{Acknowledgement}

This work is supported by the Hungarian National Office for Research and Technology through grants TECH_08_2/2-20080088, OM-0239/2008, which is gratefully acknowledged. The work reported in the paper has also been developed in the framework of the project "Talent care and cultivation in the scientific workshops of BME" project. This project is supported by the grant TÁMOP-4.2.2.B-10/1-2010-0009.

\section{András Mihály}

Department of Control for Transportation and Vehicle Systems, Budapest University of Technology and Economics,

Stoczek u. 2., H-1111 Budapest, Hungary

e-mail: mihaly.andras@mail.bme.hu

\section{Balázs Németh}

Péter Gáspár

Computer and Automation Research Institute, Hungarian Academy of Sciences, Kende u. 13-17., H-1111 Budapest, Hungary

\section{Introduction}

Conventional cruise control systems which are able to maintain steady speed have been used in the automotive industry for several decades. These systems can maintain steady speed by adjusting the propulsion force acting on the vehicle, i.e modifying the throttle status according to the disturbance acting on the vehicle. Among today's middle and premium category vehicles adaptive cruise control systems which are able to follow the preceding vehicle in a driver defined safe distance are becoming increasingly widespread. These systems use radar sensors to observe the traffic, and the intervention of the brake system is also required.

In the paper a look-ahead control method for the design of the vehicle's speed is applied, in which the road inclinations and speed limits are taken into consideration, see [9]. In this method information about the current and oncoming road sections such as speed limits and road slopes are required. With the consideration of road information the selected speed can be in coherence with the oncoming road, thus speed can be reduced in advance of a slope or a speed limit. By selecting an optimal speed for the vehicle unnecessary accelerations and brakings can be reduced. This results in moderated energy and fuel consumption and in addition, the wearing of the brake system is also reduced. These attributes of the look-ahead system also benefits the maintenance cost of the vehicle. Several look-ahead methods have already been proposed, see [3, 4, 10, 11].

The speed proposed by the look-ahead control system can differ from the speed which is natural for a human driver. The driver's speed selection depends on limited and inaccurate visual information about the oncoming road. The driver's behavior in term of velocity selection is depending on instantaneous effects, such as disturbances acting on the vehicle, the traffic situations etc.

The paper focuses on a multi-criteria look-ahead control design considering objectives such as the minimization of energy and consumption as well as the incorporation of the driver behavior. The results of the design are validated in a CarSim simulation environment.

This paper is organized as follows. In Section 2 a speed 
tracking driver model is introduced along with the driver simulation environment. In Section 3 the optimization criteria such as energy optimization and the driver behavior adaptation are detailed. In Section 4 the components of the multi-criteria optimization are presented. In Section 5 the operation of the lookahead controller is presented in a real data simulation example. Finally, Section 6 contains some concluding remarks.

\section{Driver model}

For further analysis, a driver model is used to capture the behavior of the driver in terms of following the desired speed. [5] developed a hybrid driver model, in which the discrete event system theory was combined with the classical control theory. In the driver model visual perception was divided into two classes, i.e., the traffic-relevant and the vehicle-relevant factors. Queuing networks were particularly suited for modeling parallel activities, while symbolic models had particular strength in generating a person's actions in specific task situations. The neural network model, fuzzy logic and genetic algorithm approaches of the driver models are also widely used. see e.g., [6]. The linearized model used in this paper [1] assumes that the driver perceives only forward speed, and the dynamic model of the vehicle is known. The architecture of the driver model has been presented in [7]. The two main element of the model is the transfer function $Y_{u}$ representing the driver and the transfer function representing the vehicle dynamics. The transfer function of the driver is as follows:

$$
Y_{u}=K_{u}\left(\frac{1}{s}+T_{L}\right) e^{-s \tau}
$$

A representative set of driver parameters used for the simulation: $K_{u}=0.3 ; T_{L}=12 ; \tau=1.7$. These parameters are approximated by the values gained from driving simulator studies.

\section{Driver simulation environment}

A real-time simulation environment has been built in our laboratory. The longitudinal driver model can be identified with measurements in a real-time simulation environment, in which the Driving Simulator of CarSim is used. Figure 1 shows the architecture of the simulator with a real car connected to a simulation environment. For simulation purposes, the control of the vehicle's communication network has been taken over by the simulator unit.

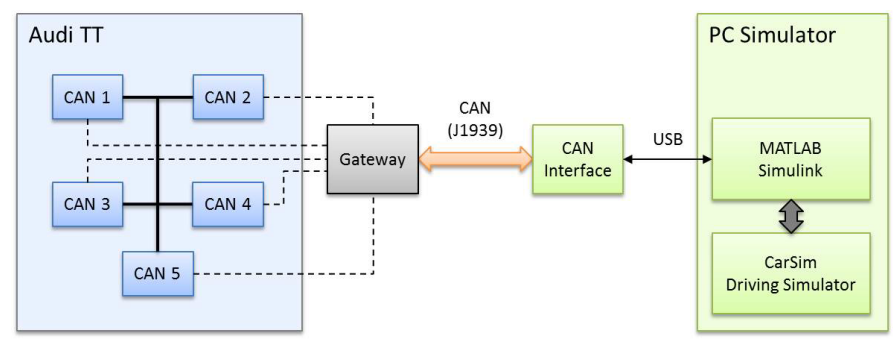

Fig. 1. Architecture of driving simulator

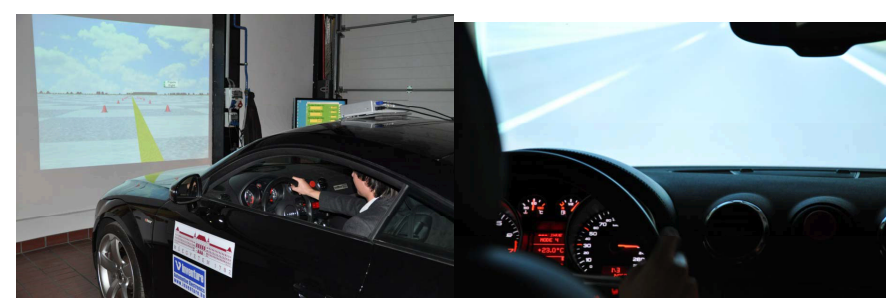

Fig. 2. The simulator environment

The simulation environment consists a vehicle incorporating HMI (Human Machine Interface) functions and a simulator application based on a PC. The simulation is running under MATLAB/Simulink environment using the differential equation solvers of MATLAB. The high-accuracy validated software of CarSim implements the physical model of the vehicle and the simulation environment. The results are projected in front of the vehicle in real-time graphics. Various driver can be seated in the vehicle to control the accelerator and brake pedals, the steering wheels and the gear lever. The driving experience is close to real life driving experience, however, the feel of acceleration is lacking since the vehicle is not moving during the simulation.

An illustration is shown in Figure 2

One of the main advantage of the system is that in principle any signals can be monitored during the simulations, even signals which are difficult if not impossible to measure in real life tests. The results of the simulations can be used to set up longitudinal driver models for different type of drivers, i.e aggressive or calm, awake or tired etc. These driver attitudes can result in different behavior in respect of following the speed limit or reacting on a big disturbance acting on the vehicle. For example, an aggressive driver may exceed the speed limit more often and use the break and accelerator pedal more intensely than a calm driver. Also, a tired driver may react slowly to the disturbances acting on the vehicle, thus it can slow down more on uphill section and can exceed the speed limit on a slope. Thus, with the use of the simulation environment it is possible to identify a set of parameters which correspond to an ordinary driver. These parameters can be adopted in the vehicle model described in Section 2 .

\section{Optimization factors for multi-criteria speed design}

\subsection{Energy optimization using road slopes}

The relationship between the energy optimal speed and the road inclinations was introduced in [7,8]. Thus, in this paper the detailed calculation of the optimal velocity is omitted, only the main results are summarized. The assumption is, that the path of the vehicle can be divided into $n$ number of sections using $n+1$ number of points, see Figure 3

The rates of the inclinations of the road and those of the speed limits are assumed to be known at the endpoints of each section. The speed at section point $j$ should reach a predefined reference speed $v_{r e f, j} j \in[1, n]$, which is the speed limit on the section, while the momentary value of the speed limit must also be taken 
Fig. 3. Division of predicted road

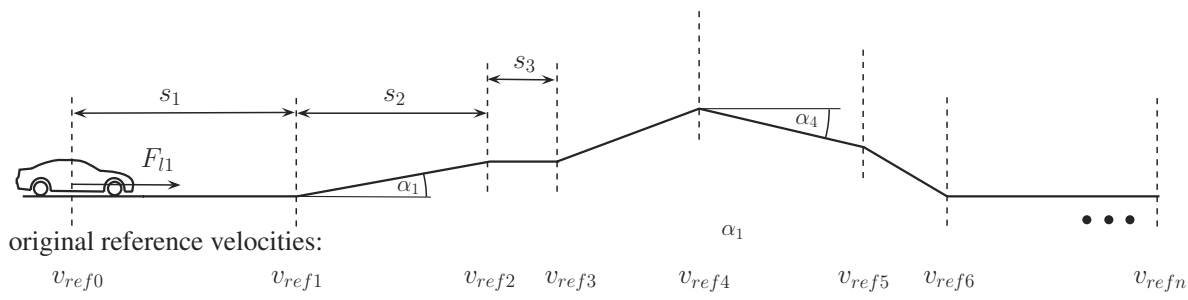

modified reference velocity:

into consideration in the following form: $\dot{\xi}_{0}^{2} \rightarrow v_{r e f, 0}^{2}$. The speed of the $n^{\text {th }}$ section point is the following:

$$
\dot{\xi}_{n}^{2}=\dot{\xi}_{0}^{2}+\frac{2}{m} s_{1} F_{l 1}-\frac{2}{m} \sum_{i=1}^{n} s_{i} F_{d i}
$$

where $F_{d i}$ is the disturbance force originating from the road slopes and other disturbances such as rolling resistance, aerodynamic forces.

After adding weight $Q$ to the momentary speed and weights $\gamma_{1}, \gamma_{2}, \ldots, \gamma_{n}$ to the reference speeds of the road sections in advance $\left(\gamma_{1}+\gamma_{2}+\ldots+\gamma_{n}+Q=1\right)$, the following formula is yielded for the optimal vehicle velocity:

$$
\dot{\xi}_{0}^{2}+\frac{2 s_{1}}{m}(1-Q)\left(F_{l 1}-F_{d 1, o}\right)=\vartheta
$$

where value $\vartheta$ depends on the predicted road slopes, the reference velocities and the prediction weights:

$$
\begin{gathered}
\vartheta=Q v_{r e f, 0}^{2}+\sum_{i=1}^{n} \gamma_{i} v_{r e f, i}^{2}+\frac{2}{m}\left(\sum_{i=1}^{n}\left(s_{i} F_{d i, r} \sum_{j=i}^{n} \gamma_{j}\right)\right. \\
+\sum_{i=2}^{n}\left(s_{i} F_{d i, o} \sum_{j=i}^{n} \gamma_{j}\right) \\
\lambda=\sqrt{\vartheta-2 s_{1}(1-Q)\left(\ddot{\xi}_{0}+g \sin \alpha\right)}
\end{gathered}
$$

where

$$
\begin{gathered}
\vartheta=Q v_{r e f, 0}^{2}+\sum_{i=1}^{n} \gamma_{i} v_{r e f, i}^{2}+\frac{2}{m}\left(\sum_{i=1}^{n}\left(s_{i} F_{d i, r} \sum_{j=i}^{n} \gamma_{j}\right)\right. \\
+\sum_{i=2}^{n}\left(s_{i} F_{d i, o} \sum_{j=i}^{n} \gamma_{j}\right)
\end{gathered}
$$

Equation (5) shows that the modified reference speed $\dot{\xi}_{0}$ depends on weights $Q$ and $\gamma_{i}$.

\subsection{Tracking the behavior of the driver}

The driver's visual perception of the road ahead is much shorter than the road known by the automatic system, and the human driver can only approximate the road inclinations. Thus it is assumed that in the speed selection process the driver tries to follow the regulated maximum speed and only considers instantaneous effects such as disturbances acting on the vehicle.

For the consideration of the driver behavior, the speed selection algorithm is modified in such a way that weight $Q$ is substituted for by the weight that the driver would have used at the same road section. The calculation of the driver weight $Q_{d}$ is as follows: by ignoring the road information, the values of $\gamma_{i}$ are presumed to be zero. Thus in the mapping of the drivers' possible weight selection, the problem is simplified to the calculation of the constantly changing $Q_{d}$ weight, which can be calculated on-board during the journey of the vehicle. Assuming that the vehicle dynamics and the driver's function are known along with the actual reference speed and the road slope, it is possible to calculate the speed which the driver would have chosen in the presence of the actual disturbances. Then, by measuring the actual acceleration of the vehicle, it is possible to calculate weighting function $Q_{d}$ which the driver would use if it were an automatic system. Note that by this method, the driver's behavior in terms of following the actual reference speed is mapped.

The calculation method is derived as follows:

After organizing equation (5) and substituting $\ddot{\xi}_{0}=\left(F_{l}-\right.$ $\left.F_{d, o}-F_{d 1, r}\right) / m$ the following equation is derived to determine the speed of the vehicle:

$$
\vartheta=\dot{\xi}_{0}^{2}+2 s_{1}(1-Q)\left(\ddot{\xi}_{0}+g \sin \alpha\right)
$$

Next equations (6) and (7) are combined, assuming $\gamma_{i}$ to be zero:

$$
\dot{\xi}_{0}^{2}+2 s_{1}(1-Q)\left(\ddot{\xi}_{0}+g \sin \alpha\right)=Q v_{r e f, 0}^{2}
$$

Rearranging the equation, weight $Q=Q_{d}$ can be expressed as follows:

$$
Q_{d}=\frac{\dot{\xi}_{0}^{2}+2 s_{1} \ddot{\xi}_{0}+2 s_{1} g \sin \alpha}{v_{r e f, 0}^{2}+2 s_{1} \ddot{\xi}_{0}+2 s_{1} g \sin \alpha}
$$

where $\dot{\xi}, \ddot{\xi}$ are calculated with the above driver model, $\alpha$ and $v_{\text {ref }, 0}$ are road information assumed to be known.

The automatic look-ahead system can be modified by selecting the $Q_{d}$ values calculated by using the driver model. In this way, the optimization process of the look-ahead system can adopt the $Q_{d}$ values that the driver may have used in the same section of the route in order to determine $\gamma_{i}$ weights. By this method, the speed profile and the traveling time will be closer to that of the human driver. The consideration of the road slope will still be captured in the cruise control with a smaller weight, thus the energy consumption will be lower than those with a human driver's. 


\section{Multi-criteria optimization}

The aim of this section is to find an optimal speed $\dot{\xi}_{0}$, which guarantees the minimization of control force (fuel consumption) and the difference of the speed proposed by the driver model and the look-ahead system. The fulfillment of these performances individually results in different $Q, \gamma_{i}$ weights according to equation (5).

\subsection{Minimization of control force}

By using equation (5) the longitudinal force $\left(F_{l 1}\right)$ can be expressed as the linear function of prediction weights:

$$
F_{l 1}=\beta_{0}(Q)+\beta_{1}(Q) \gamma_{1}+\ldots+\beta_{n}(Q) \gamma_{n}
$$

where $\beta_{i}$ are the coefficients of $\gamma_{i}$, and they depend on weight $Q$.

In the case of the minimization of control force $\left|F_{l 1}\right| \rightarrow$ Min! must be guaranteed. In practice, however, the $F_{l 1}^{2} \rightarrow$ Min! optimization is used because of the simpler numerical computation. This minimization problem is solved by the transformation of the quadratic form with the following constrains:

$$
\bar{F}_{l 1}^{2}\left(\bar{Q}, \bar{\gamma}_{i}\right)=\left(\bar{\beta}_{0}(\bar{Q})+\bar{\beta}_{1}(\bar{Q}) \bar{\gamma}_{1}+\ldots+\bar{\beta}_{n}(\bar{Q}) \bar{\gamma}_{n}\right)^{2}
$$

where $0 \leq \bar{Q}, \bar{\gamma}_{i} \leq 1$ and $\bar{Q}+\sum \bar{\gamma}_{i}=1$. This task is a nonlinear optimization problem because of the weights. With fixed weights (11) becomes a quadratic optimization problem. Its solution is found in [2].

\subsection{Minimization of the difference between the driver's speed and the proposed speed}

Another optimization criterion of the vehicle cruise control is the minimization of the difference between the selected speed and the speed that the driver would have chosen at the current road section. This criterion is important for the comfortable travel for the driver and passengers. Therefore the difference between the momentary speed and the driver speed must be minimized, i.e.,

$$
\left|v_{\text {driver }}-\dot{\xi}_{0}\right| \rightarrow \operatorname{Min} !
$$

This optimization criterion can be fulfilled if $Q=Q_{d}$ is selected and the road inclinations are ignored, i.e $\breve{Q}=Q_{d}$ and $\breve{\gamma}_{i} \equiv 0, i \in$ $[1, n]$, where $Q_{d}$ is given is given in equation 9

\subsection{Simulation example on undulating road and with speed limit}

For the illustration of the different velocity profile set by the driver and the automatic system a simulation was performed on a section with 5 percent of road inclination and a speed limit of $80 \mathrm{~km} / \mathrm{h}$, see Figure 4 . It can be observed, that the velocity set by the driver increases on the slope and falls back on the uphill, whereas the conventional cruise control manages to follow the reference velocity with little deviation. The operation of the look-ahead system is also well demonstrated. The vehicle decreases it's velocity before the slope because the controller

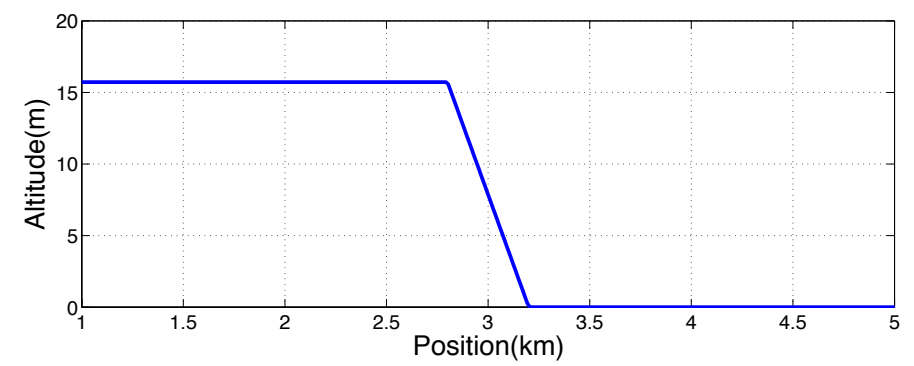

(a) Road altitude

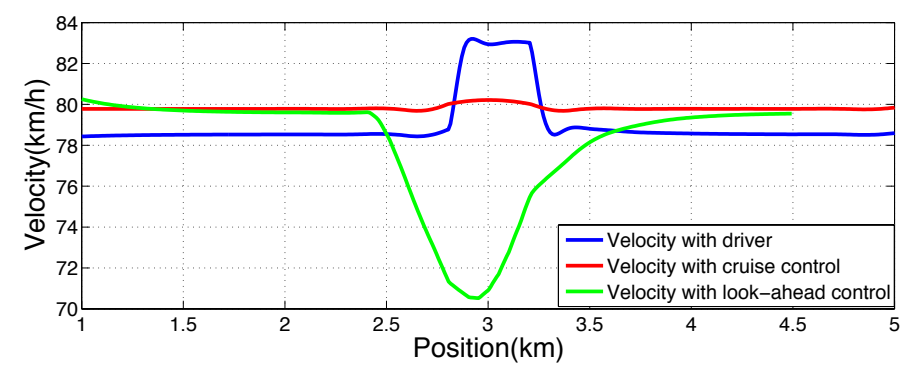

(b) Velocity of the vehicle

Fig. 4. Driver and cruise control systems on a hilly road

reduces the required longitudinal force, knowing that the slope will accelerate the vehicle.

The situation is similar when there is a change in the speed limit on the road, see Figure 5. The controlled vehicle with look-ahead system can decrease it's velocity in advance, while the driver decelerates more intensely. Comparing these velocity profiles it is obvious that the look-ahead system's velocity differs greatly from that set by the driver. This results in a more efficient travel, however, the driver and the passengers of the vehicle may feel uncomfortable because of the unusual velocities that the look-ahead system follows. In case of heavy traffic with other vehicles following velocity profiles closer to that suggested by the driver model or the regular cruise control, this unusual velocity profile may interfere with the traffic environment. For this reason, it is reasonable to capture the driver's behavior in the velocity selection process of the look-ahead system.

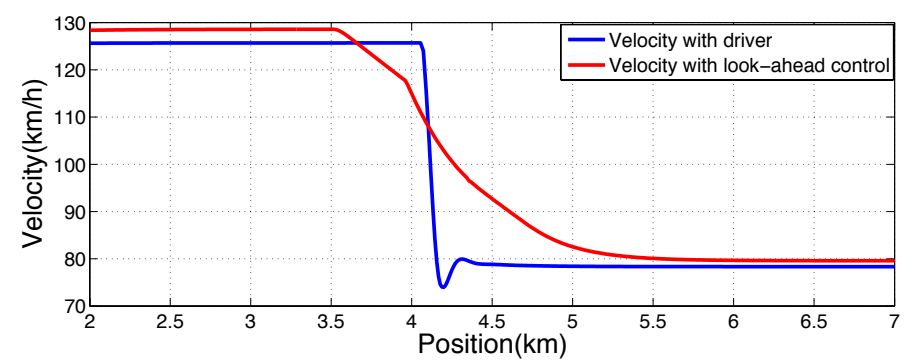

Fig. 5. Driver and look-ahead system with changing speed limit

The goal of the multi-criteria optimization is to find a good compromise between energy efficient travel and driving comfort. 


\subsection{Multi-criteria optimization}

The two performances listed in 4.1 and 4.2 results in different $Q$ and $\gamma$ weights. During the multi-criteria design an additional tuning of the weights is necessary to realize a good trade-off between energy optimization and traveling comfort. In the proposed method two further performance weights $R_{1}$ and $R_{2}$ are introduced for this reason. Performance weight $R_{1}\left(0 \leq R_{1} \leq 1\right)$ is related to the importance of the minimization of the longitudinal control force $F_{l 1}$, while performance weight $R_{2}\left(0 \leq R_{2} \leq 1\right)$ is related to the importance of the driver's behavior adaptation. There is a constraint according to the performance weights $R 1+R 2=1$. Thus the performance weights, which guarantee a balance between the optimizations tasks, are calculated in the following expressions:

$$
\begin{aligned}
Q & =R_{1} \bar{Q}+R_{2} \breve{Q} \\
\gamma_{i} & =R_{1} \bar{\gamma}_{i}+R_{2} \breve{\gamma}_{i}=R_{1} \bar{\gamma}_{i}, i \in[1, n]
\end{aligned}
$$

Based on the optimization method the reference speed $\lambda$ of the vehicle is calculated.

\subsection{Realization of the method}

The control system can be realized in three steps.

Based on the three optimization tasks the weighting factors are calculated in the first step. Then using (13) $Q$ and $\gamma_{i}$ are calculated. Finally, the reference speed is calculated, see equation (5).

In the second step the longitudinal control force of the vehicle $\left(F_{l 1}\right)$ is calculated. It is a robust design step, in which $H_{\infty}$ design method is applied. The result is the required longitudinal force, which could be positive and negative forces as well, therefore the driving and braking systems are also actuated.

In the third step the real physical inputs of the system, such as the throttle, the gear position and the brake pressure are generated by the low-level controller. Figure 6 shows the architecture of the low-level controller.

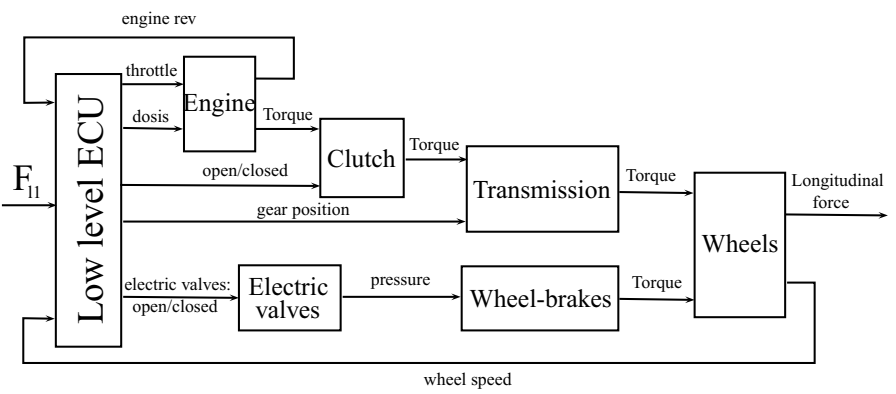

Fig. 6. Architecture of the low-level controller

In the proposed method the steps are separated from each other. Thus the reference signal unit can be designed and produced independently of automobile suppliers and only a few vehicle data are needed. The independent implementation possibility is an important advantage in practice.

\section{Simulation results}

In this section the optimization method is analyzed through real data motorway simulation in the CarSim environment. The terrain characteristics and geographical information are those of the M1 Hungarian highway between Tatabánya and Budapest in a 56- $\mathrm{km}$-long section with several uphill and downhill slopes. The regulated maximum speed is $130 \mathrm{~km} / \mathrm{h}$, but the road section contains other speed limits as well (e.g. $80 \mathrm{~km} / \mathrm{h}$ or $100 \mathrm{~km} / \mathrm{h}$ ). The vehicle used for the simulation is an F-Class sedan with a $300 \mathrm{~kW}$ engine, meeting the EURO 4 emission standards.

For the validation of the optimization methods listed in Section 3 three simulations were carried out.

In the first simulation the behavior of the driver was demonstrated using the driver model described in Section 2. In the second simulation the look-ahead control detailed in Section 3 was implemented in order to minimize the actuated energy of the vehicle. Finally, a simulation was performed using the multicriteria optimization method.

The operation of the multi-criteria controller is as follows: with the driver model detailed in Section 2, the speed of the driven vehicle is defined at each sample time, based on the driver model's dynamics and the actual disturbances (slope angle, speed regulations, drag disturbance and rolling resistance) acting on the vehicle. From the driver speed weighting function $\breve{Q}$ is calculated as detailed in Section 3.2 representing the behavior of the driver. At the same time energy optimal velocity considering forward road information is also calculated as detailed in section 3.1 and 4.1 as well as the corresponding $\bar{Q}$ and $\bar{\gamma}_{i}$ weights. With the use of the multi-criteria tuning weights $R_{1}$ and $R_{2}$ the final weights $Q$ and $\gamma_{i}$ are given as listed in 4.4 . Thus the reference speed for the vehicle can be calculated using (5). The realization of the speed controller is detailed in Section 4.5 .

In Figure 7(a) the terrain characteristic of the motorway section is shown. In Figure 7(b) the speed profiles are shown resulting from the driver and the different control methods. It can be observed that the driver tends to follow different speed profile relative to that given the look-ahead control. The velocity of the vehicle is more undulating and the driver may exceed the speed limit traveling on heavy slope, while the velocity of the vehicle falls back on uphill sections. This is due to the fact that the driver does not have information about the oncoming road conditions, while the look-ahead control control method involves future disturbances in the speed design. As expected, the multicriteria design provides a well balanced compromise among the velocity profile of the driver and the automatic system. In Figure 7) (c) the actuated energy (brake and propulsion) of the vehicle is shown during the travel.

It is well demonstrated, that the behavior of the driver results in larger amount of actuated energy compared to that of the lookahead control, while the multi-criteria design can achieve almost the same result as the energy optimal control. The same conclusion can be drawn for the fuel consumption of the vehicle. The 


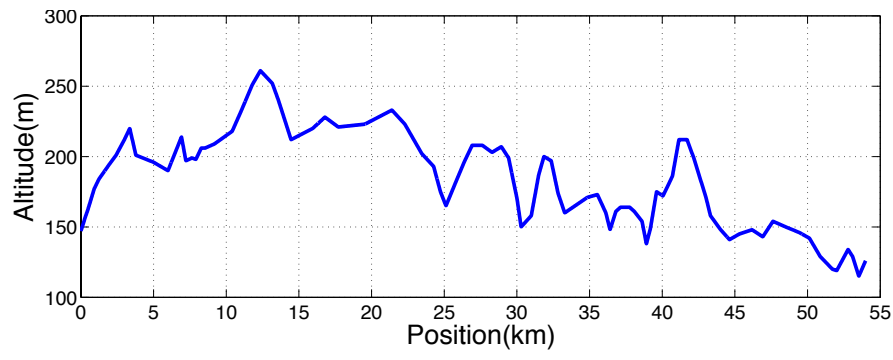

(a) Terrain characteristics of the path

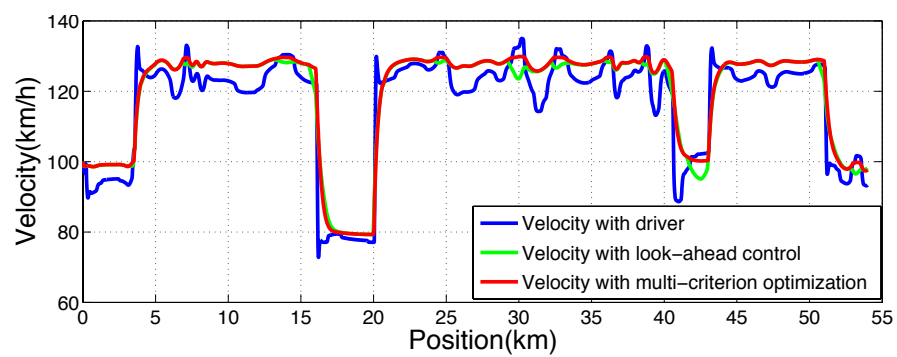

(b) Speed profiles of different methods

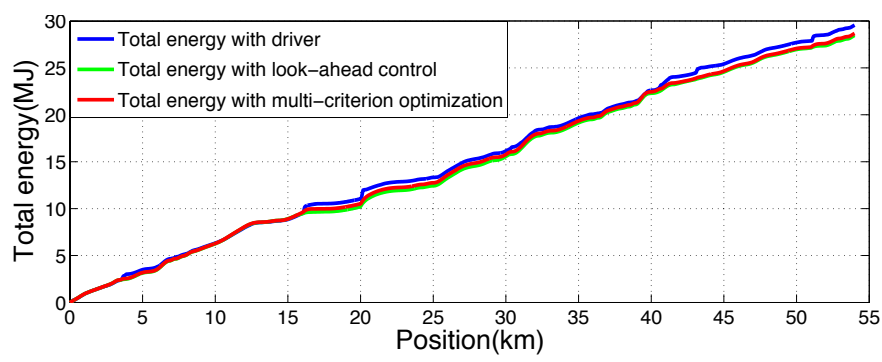

(c) Total actuated energy of different methods

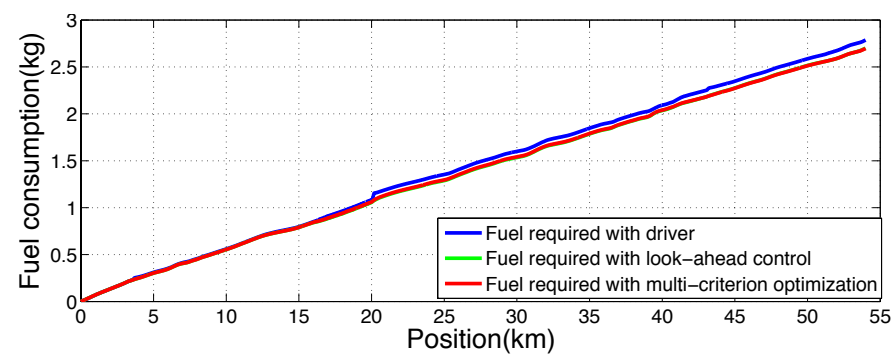

(d) Fuel consumption of different methods

Fig. 7. Results of the multi-criteria design compared to other methods

abrupt speed changes by the driver result in harder and more frequent brake use and intense accelerations, which is the cause of the larger fuel consumption. In addition travel time is more than one minute longer than in the case of energy optimized and multi-criterion travel.

\section{Conclusion}

The paper has presented a multi-criteria design for vehicle speed control considering energy consumption and driver behavior. It has been demonstrated that with multi-criteria design a satisfactory compromise can be achieved between the two criteria of fuel economy and passenger comfort. The main novelty of the paper is the incorporation of the driver speed selection behavior in the automatic look-ahead control system, which can enhance the comfort level of the driver and the passengers by adjusting the speed to be closer to that of a human driver.

\section{References}

1 Cacciabue PC, Modelling Driver Behaviour in Automotive Environments, Springer; London, 2007, DOI 10.1007/978-1-84628-618-6

2 Coleman TF, Li Y, A reflective newton method for minimizing a quadratic function subject to bounds on some of the variables, SIAM Journal on Optimization, 6(4), (1996), 1040-1058, DOI 10.1137/S1052623494240456

3 Hellström E, Ivarsson M, Åslund J, Nielsen L, Look-ahead control for heavy trucks to minimize trip time and fuel consumption, Control Engineering Practice, 17(2), (2009), 245-254, DOI 10.1016/j.conengprac.2008.07.005

4 Ivarsson M, Åslund J, Nielsen L, Look ahead control - consequences of a non-linear fuel map on truck fuel consumption. Part D, In:, Proceedings of the Institution of Mechanical Engineers, 2009, pp. 1223-1238.

5 Kiencke U, Majjad R, Kramer S, Modeling and performance analysis of a hybrid driver model, Control Engineering Practice, 7, (1999), 985-991, DOI 10.1016/S0967-0661(99)00053-2

6 Liu Y, Queueing network modeling of human performance of concurrent spatial and verbal tasks. Part A, Systems and Humans, In: IEEE Transactions on Systems Man and Cybernetics, Vol. 27, 1997, pp. 195-207.

7 Mihály A, Németh B, Gáspár P, Velocity selection by a human driver compared to look-ahead controll, Periodica Polytechnica, (2012).

8 Nemeth B, Gaspar P, Lpv-based control design of vehicle platoon considering road inclinations, 18th IFAC World Congress, 2011, In:.

9 Nemeth B, Gaspar P, Road inclinations in the design of lpv-based adaptive cruise control, 18th IFAC World Congress, 2011, In:.

10 Nouveliere L, Braci M, Menhour L, Luu HT, Fuel consumption optimization for a city bus, UKACC Control Conference, 2008, In:.

11 Sahlholm P, Johansson KH, Road grade estimation for look-ahead vehicle control using multiple measurement runs, Control Engineering Practice, (2009), in press 